\title{
Priorities for Greening and the Sustainable Development of OECD Member Countries and Ukraine: a Comparative Analysis
}

\author{
Olena Dovgal (iD) https://orcid.org/0000-0003-3219-9731 \\ Dr.Sc. of Economics, Professor (full) at the V.N. Karazin Kharkiv National University \\ Kharkiv, Ukraine, e-mail: e.dovgal@karazin.ua
}

Nataliia Goncharenko (iD) https://orcid.org/0000-0003-4148-5369

Ph.D. (Economics), Associate Professor at the V.N. Karazin Kharkiv National University, Kharkiv, Ukraine, e-mail: n.i.goncharenko@karazin.ua

Olena Reshetnyak (iD https://orcid.org/0000-0002-1183-302x

Ph.D. (Economics), Associate Professor at the V.N. Karazin Kharkiv National

University, Kharkiv, Ukraine, e-mail: olena.reshetnyak@karazin.ua

Georgiy Dovgal (iD https://orcid.org/0000-0002-0644-1793

Ph.D. (Economics), Associate Professor at the V.N. Karazin Kharkiv National University, Kharkiv, Ukraine, e-mail: g.dovgal@karazin.ua

Natalia Danko (iD https://orcid.org/0000-0002-2977-6641

Ph.D. (Economics), Associate Professor at the V.N. Karazin Kharkiv National University, Kharkiv, Ukraine, e-mail: n.danko@karazin.ua

\begin{abstract}
The article focuses on identifying priority areas for greening and sustainable development for OECD countries and Ukraine. They aim to achieve overall progress in the interaction between the economy and the environment. Additionally, the aim is to create prerequisites for encouraging innovation and investment to find new sources of economic growth that are compatible with ecosystems that are capable of recovering from damage. It has been demonstrated that although the global goals of greening economies are relevant for all countries, they must be tailored to the regional and national specificities, as well as each country's level of economic development.
\end{abstract}


The study used general qualitative and quantitative methods of economic research, including systematic, comparative analysis, methods of logical and statistical analysis, and index method, among others. The applied theoretical and methodological approach allowed us to identify general trends in the development of environmental factors in the OECD countries and Ukraine and their impact on economic growth. Specifically, the study analyzes the current state of affairs and perspectives for greening and sustainable development based on a comprehensive assessment of the level of greening in these economies and the relationship between the internal ecological environment - assessed using the indicators of "green growth" - and their economic development. The article also justifies priorities for greening and sustainable development and suggests practical measures for their implementation. They can serve as a basis for developing a policy of effective environmental management and elaborating a national system of environmentally friendly management and administration.

Keywords: global environmental problems, global environmental strategy, green growth, greening, priorities for greening and sustainable development

JEL: F55, O13, O44, O57, P51, Q56

\section{Introduction}

Identifying priority areas for greening and sustainable development must be based on a justified environmental strategy, which aims, first and foremost, to improve the current state of the global eco-economic space. As the environment where this environmental strategy is to be implemented is not stable, the strategy's function is to initiate well-timed changes that can increase the chances for sustainable development. Environmental strategies can be shaped at different levels: global, territorial (e.g., pan-European, pan-Asian, EU), regional (e.g., Danube valley) and national. Levels of national strategy can also be defined in terms of the national economy or business organization, namely: interdisciplinary (multi-sectoral strategy), specific (partial, limited strategies for influencing environmental and economic processes in various aspects) and functional (e.g., finance, education, innovation strategy).

In recent years, rapid and unpredictable changes in the economy have influenced the environment. Reconciling the interests of participants in socio-economic life and their long-term interest in protecting the environment at the macro level with short-term political and economic interests is becoming increasingly complex. Currently, only countries that are aware of these changes can assess their impact and take timely action caton adapt to external threats (United Nations 2018; New Atlas of the Green Economy 2019). That is why the successful implementation of an environmental strategy at the global level, as well as identifying priorities for greening and sustainable development, is largely determined not by financial means or the physical environment, but by the complex of environmental knowledge and environmental culture (Zerkalov 2013, p. 27). 
The importance of a strategic vision when justifying priority areas of sustainable development greening is due to the increasing uncertainty and awareness of environmental deterioration, increasing the speed of change concerning both the environment and the economy, strengthening the effects of globalization. This, in turn, increases the demand for strategic international partnerships, the spread of regionalism processes worldwide, and the necessity to modernize the existing international system of relations (Dovgal and Panova 2018a, pp. 380-385). All this requires new methods to justify the environmental strategy for sustainable development.

The purpose of this article is to identify the priority areas of sustainable development greening and practical measures for their implementation for OECD countries and Ukraine, taking into account regional and national specifics, as well as the economic development level of each country. All this requires new methods to justify the environmental strategy for sustainable development. Specifically, there should be a comprehensive assessment of the economic greening level by calculating its greening index, based on the relationship characteristics of its internal ecological environment with its economic development, assessed by indicators of "green growth." The applied theoretical and methodological approach allowed us to identify the general trends in the development of environmental factors in the OECD countries and Ukraine and their impact on economic growth.

\section{Literature review}

This paper is related to several strands of literature. First, this article contributes to the literature that studies the evolution of nature and society's interaction concepts based on the research of problem areas such as the cost characteristics of the land of William Petty (Hull 1899, pp. 21-38), the emergence of global environmental crises under human influence (Malthus 1798, pp. 14-29), the world economy development modelling, which provides technological progress and economic growth restraint (Meadows et al. 1972, pp. 4-12; Meadows et al. 2007, pp. 5-16), and the development of international trade, taking into account the environmental factor (Anderson and Blackhurst 1992, pp. 12-35; Esty, 1994, pp. 9-28; Daly and Farley 2010, pp. 19-24).

Currently, from the standpoint of environmental and economic policy in the economic literature, two basic generalized models have been determined: a model of the front economy, which does not consider the scale of resources consumption concerning their reserves as the determining parameters of further system development, the primary factors limiting economic development; and the concept of environmental protection, which recognizes the necessity to consider the environmental factor, which is considered a factor that limits economic development (Kazakov et al. 2009, pp. 2133; Svenningsen and Thorsen 2020, pp. 1-24).

To determine the level of environmental responsibility of countries, American ecologist Paul Ehrlich and physicist John P. Holdren (Ehrlich et al. 1977) proposed a meth- 
odological approach to assessing anthropogenic environmental impact, according to which the environmental impact is associated with the country's level of economic welfare. The development of this direction in the economic literature led to the appearance of the term' ecological footprint' as an indicator of the intensity of human negative impact on the environment, as calculated in hectares of land per capita (Wackernagel et al. 2004, pp. 293-315; Kitzes et al. 2007, pp. 1-4; Wackernagel et al. 2019).

In the scientific developments of the late twentieth century ecosystem concept is reflected in the study of assimilation potential, which implies the ability of the ecosystem to absorb harmful impurities without significant consequences for its equilibrium (Ignatov et al. 1999, pp. 32-51).

Weizsäcker, Lovins, and Lovins (1995) proposed the "Factor Four" concept, which provides a 4 -fold increase in resource productivity. They later produced "Factor Five", to achieve an $80 \%$ increase in resource efficiency, specific technologies and solutions. They also demonstrated ways of scaling these solutions and technologies globally, to change the economic system in favor of solving the problems of climate change, fresh water deficit, hunger, and waste (Weizsäcker et al. 2010).

Modern developments towards identifying and solving the problems of environmental management and the greening process are based on theoretical and methodological studies by researchers such as Colby (1989), Huber (1991), Blanc et al. (2008, pp. 251-260), among others. The sustainable development of modern civilization and the population's quality of life are directly related to insufficient energy supply (Matyushenko et al. 2015, p. 9). To solve the contradictions between economics and nature, Banerjee et al. (2016) proposed an approach to analyze policy impacts on the economy and the environment in a quantitative, comprehensive, and consistent framework.

We agree with most economic and environmental experts (Rogers et al. 2006, pp. 12-31; Podlesnaya 2012, pp. 208-212; Wiesmeth 2012, pp. 5-14; Anderson 2013, pp. 7-19; Reznikova 2016, pp. 23-26; Jackson 2017, pp. 4-49; Škrinjarić 2020, pp. $72-$ 108) that the greening process, as a tool to ensure environmental safety and achieve the sustainable development of eco-economic production systems, is necessary and the only right way to solve this problem. However, it should be noted that, at present, there is a great need to develop ecological and economic system development concepts that will sustainably develop civilization and, at the same time, ensure economic growth and ecological balance in the world.

\section{Materials and methods}

The article suggests a scientific and methodological approach to determining the hierarchical structure of the system of strategic goals, and identifying priority areas for greening and sustainable development (Figure 1).

In order to successfully implement an environmental strategy in the process of greening and sustainable development, it is important to develop a realistic vision 
of it, explain it to stakeholders in every detail, make it appealing to them, and ensure that certain actions are taken within the framework defined by this vision. At the same time, it involves choosing the overall direction of sustainable development by disclosing the system of values, and with the participants' active involvement and aptitude for risk-taking in the process of implementing an environmental strategy.

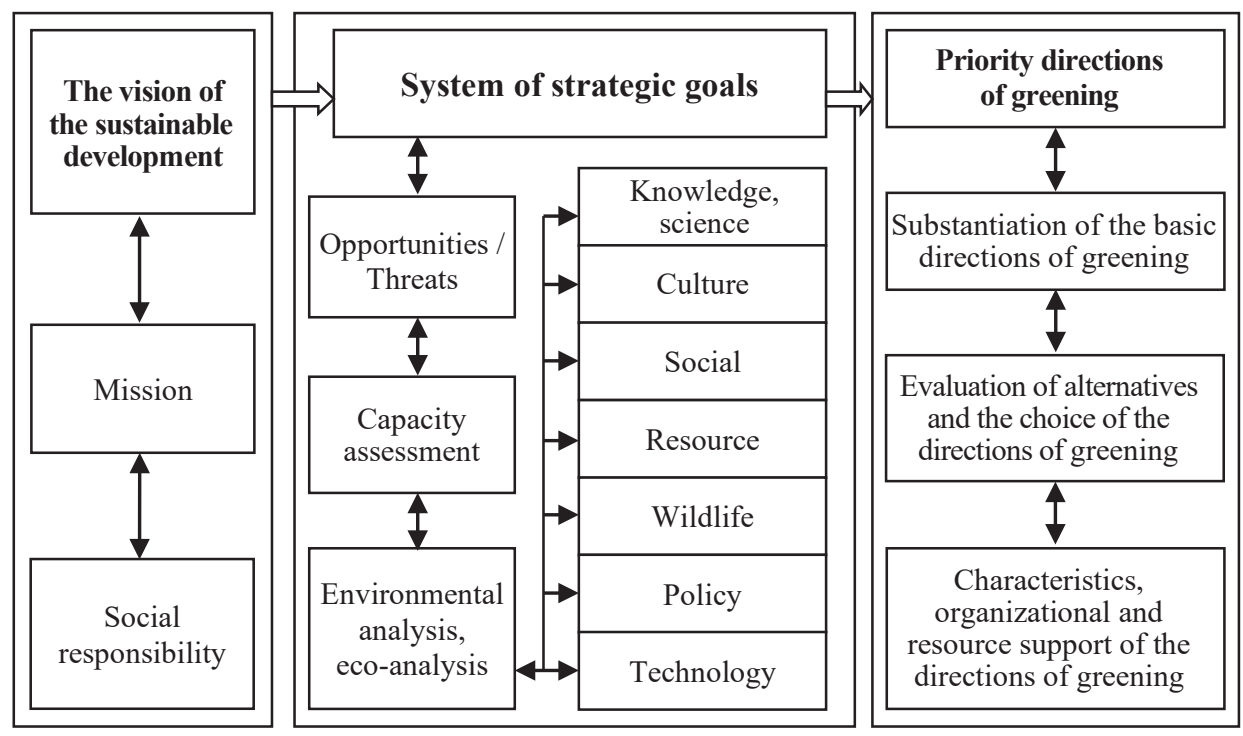

Figure 1. Determining the hierarchical structure of the system of strategic goals and identifying priority areas for greening and sustainable development

Source: Global Goalscast 2018; United Nations 2018.

The vision for sustainable development also outlines areas for innovation. Sustainable development, in line with OECD recommendations, is defined in terms of "green growth", namely "the introduction of means to foster economic growth and development as well as ensure the preservation of the natural assets on which the welfare of mankind depends" (OECD 2018). Green growth itself should be a catalyst for investment and innovation, which will lay the foundations for sustainable development, creating new economic opportunities. In formulating the principles of green growth, the OECD relied on a number of analytical studies and political efforts to ensure sustainable development. That is why these environmental and economic growth benchmarks have been chosen in our work to justify our vision of priority areas for sustainable development (United Nations 2018; Sustainable Development Report 2019; United Nations 2019; World Resources Institute 2018).

The system of strategic goals for greening and sustainable development has a hierarchical structure that we have characterized as follows: the upper level is a system of global greening goals; the second one includes a system of national goals; the third one contains a system of goals in specific areas of greening. Sustainable development 
and greening are achieved by balancing various levels of certain aspects of economic, environmental and social policies reflected in their goals, with a focus on the conservation and restoration of ecosystems in the process of economic development. Thus, they should be directed at developing and implementing the resource-efficient management of sustainably developing production and consumption (Dovgal and Panova 2018b, pp. 109-114). Global sustainable development goals in the context of tendencies and prospects of aggravating global environmental problems are summarized and described in Table 1.

Table 1. Global sustainable development goals

\begin{tabular}{|c|c|}
\hline Global environmental problems & Global goals of the greening process \\
\hline \multirow[t]{2}{*}{$\begin{array}{l}\text { The impact of harmful emissions from modern } \\
\text { industry exacerbates the environment }\end{array}$} & $\begin{array}{l}\text { Reducing the economy's carbon intensity and en- } \\
\text { hancing its resource efficiency by increasing envi- } \\
\text { ronmental friendliness and resource productivity }\end{array}$ \\
\hline & Conserving natural resources \\
\hline $\begin{array}{l}\text { The consumption of natural resources increas- } \\
\text { es, which can lead to their scarcity and the re- } \\
\text { duction of biodiversity }\end{array}$ & $\begin{array}{l}\text { Increasing economic opportunities by taking ap- } \\
\text { propriate measures for long-term use and resto- } \\
\text { ration of natural resources }\end{array}$ \\
\hline $\begin{array}{l}\text { The environmental impact of industry leads } \\
\text { to the deterioration of health and environ- } \\
\text { mental aspects of the quality of life of the } \\
\text { world's population }\end{array}$ & $\begin{array}{l}\text { Improving environmental aspects of the quality } \\
\text { of life }\end{array}$ \\
\hline
\end{tabular}

Source: Global Goalscast, 2018; United Nations, 2018.

To identify priorities for greening and sustainable development, we have comprehensively assessed how green the economy of a country is by calculating the greening index based on the relationship between its internal ecological environment and economic development, measured in terms of "green growth" indicators proposed by the OECD (OECD 1990-2018). Since the OECD database on "green growth" generates data and figures for the 1990-2018 period over a wide range (128 indicators for 46 OECD member countries, 153 non-OECD countries, and some other groups of countries), this study has used 17 key indicators for the 14 leading OECD countries according to these indicators, plus Ukraine (Table 2). 
Priorities for Greening and the Sustainable Development of OECD Member Countries and Ukraine...

Table 2. Green Growth Indicators (OECD) used in the study

\begin{tabular}{|c|c|c|c|c|}
\hline \multicolumn{3}{|r|}{ Variable } & Unit & Legend \\
\hline \multirow{9}{*}{$\begin{array}{l}\text { Environmen- } \\
\text { tal and re- } \\
\text { source pro- } \\
\text { ductivity }\end{array}$} & $\begin{array}{l}\mathrm{CO}_{2} \text { Pro- } \\
\text { ductivity }\end{array}$ & $\begin{array}{l}\text { Production-based } \mathrm{CO}_{2} \text { productivity, GDP per } \\
\text { unit of energy-related } \mathrm{CO}_{2} \text { emissions }\end{array}$ & $\begin{array}{l}\text { Number, } \\
2010\end{array}$ & $\mathrm{X}_{1}$ \\
\hline & \multirow[t]{3}{*}{$\begin{array}{l}\text { Energy } \\
\text { produc- } \\
\text { tivity }\end{array}$} & $\begin{array}{l}\text { Energy intensity, Total primary energy supply } \\
\text { per capita }\end{array}$ & $\begin{array}{l}\text { Tonnes of oil } \\
\text { equivalent } \\
\text { (toe) }\end{array}$ & $\mathrm{X}_{2}$ \\
\hline & & $\begin{array}{l}\text { Renewable electricity, \% total electricity gen- } \\
\text { eration }\end{array}$ & Percentage & $\mathrm{X}_{3}$ \\
\hline & & $\begin{array}{l}\text { Non-energy material productivity, GDP per } \\
\text { unit of domestic material consumption }\end{array}$ & $\begin{array}{l}\text { US dollars } \\
\text { per kilogram, } \\
2010\end{array}$ & $\mathrm{X}_{4}$ \\
\hline & \multirow{5}{*}{$\begin{array}{l}\text { Non-ener- } \\
\text { gy material } \\
\text { produc- } \\
\text { tivity }\end{array}$} & Biomass, \% of domestic material consumption & Percentage & $\mathrm{X}_{5}$ \\
\hline & & $\begin{array}{l}\text { Non-metallic minerals, \% of domestic materi- } \\
\text { al consumption }\end{array}$ & Percentage & $\mathrm{X}_{6}$ \\
\hline & & Metals, \% of domestic material consumption & Percentage & $\mathrm{X}_{7}$ \\
\hline & & Municipal waste generated, kg per capita & $\begin{array}{l}\text { Kilograms } \\
\text { per capita }\end{array}$ & $\mathrm{X}_{8}$ \\
\hline & & $\begin{array}{l}\text { Municipal waste recycled or composted, } \\
\% \text { treated waste }\end{array}$ & Percentage & $\mathrm{X}_{9}$ \\
\hline $\begin{array}{l}\text { Natural asset } \\
\text { base }\end{array}$ & $\begin{array}{l}\text { Forest re- } \\
\text { sources }\end{array}$ & Forest resource stocks & $\begin{array}{l}\text { Cubic me- } \\
\text { ters, Millions }\end{array}$ & $X_{10}$ \\
\hline \multirow{3}{*}{$\begin{array}{l}\text { Environmen- } \\
\text { tal dimen- } \\
\text { sion of quali- } \\
\text { ty of life }\end{array}$} & \multirow{3}{*}{$\begin{array}{l}\text { Exposure } \\
\text { to envi- } \\
\text { ronmental } \\
\text { risks }\end{array}$} & $\begin{array}{l}\text { Mean population exposure to current air } \\
\text { quality standards for fine particulate matter } \\
\left(\mathrm{PM}_{2.5}\right)\end{array}$ & $\begin{array}{l}\text { Micrograms } \\
\text { per cubic } \\
\text { meter }\end{array}$ & $\mathrm{X}_{11}$ \\
\hline & & $\begin{array}{l}\text { Mortality from exposure to ambient quali- } \\
\text { ty standards for fine particulate matter }\left(\mathrm{PM}_{2.5}\right)\end{array}$ & $\begin{array}{l}\text { Per } \\
1,000,000 \\
\text { inhabitants }\end{array}$ & $\mathrm{X}_{12}$ \\
\hline & & $\begin{array}{l}\text { Welfare costs of premature mortalities from } \\
\text { exposure to ambient quality standards for } \\
\text { fine particulate matter }\left(\mathrm{PM}_{2.5}\right) \text {, GDP equiva- } \\
\text { lent }\end{array}$ & Percentage & $\mathrm{X}_{13}$ \\
\hline \multirow{4}{*}{$\begin{array}{l}\text { Economic } \\
\text { opportuni- } \\
\text { ties and poli- } \\
\text { cy responses }\end{array}$} & \multirow{2}{*}{$\begin{array}{l}\text { Technol- } \\
\text { ogy } \\
\text { and in- } \\
\text { novation: } \\
\text { Patents }\end{array}$} & $\begin{array}{l}\text { Development of environment-related tech- } \\
\text { nologies, \% all technologies }\end{array}$ & Percentage & $\mathrm{X}_{14}$ \\
\hline & & $\begin{array}{l}\text { Development of environment-related tech- } \\
\text { nologies, inventions per capita }\end{array}$ & Number & $\mathrm{X}_{15}$ \\
\hline & \multirow{2}{*}{$\begin{array}{l}\text { Environ- } \\
\text { mental } \\
\text { taxes and } \\
\text { transfers }\end{array}$} & Environmentally related taxes, \% GDP & Percentage & $\mathrm{X}_{16}$ \\
\hline & & $\begin{array}{l}\text { Environmentally related taxes, \% total tax } \\
\text { revenue }\end{array}$ & Percentage & $\mathrm{X}_{17}$ \\
\hline
\end{tabular}

Source: OECD 1990-2018.

The study uses the index method, which makes it possible to assess how close (far) each of the 14 countries is to the benchmark (the best value of all analyzed countries), which is taken as $100 \%$. The following parameters are then converted as a percentage using the formulas (Yashalova 2015, pp. 67-75; Yashalova and Ruban 2016, pp. 219-237): 


$$
K_{i}=\frac{X_{i}}{X_{\max }} \cdot 100 \text { or } \quad K_{i}=\frac{X_{\min i}}{X_{i}} \cdot 100
$$

where $i$ is the country's number; $X_{i}$ is the parameter value for the $i$-th country; $X_{\max }$, $X_{\min }$ is the best parameter value; and $K_{i}$ is the assessment of the development level of the $i$-th country using the relevant parameter.

Based on the specific values of all the indicators for each component of the greening process in a certain country, the average value of its greening index (GI) is determined using the formula (Yashalova 2015, pp. 67-75):

$$
\mathrm{GI}=\frac{1}{n} \sum_{i=1}^{n} K_{i}
$$

The comparison of the environmental status of countries using a rating scale with a range from 0 to 100 (thus, the higher the Greening Index (GI) is, the higher the level of environmental status is) with the indicator describing economic development (average annual GDP growth rate (Knoema Corporation 2018)) provides a matrix to identify priority areas for greening and sustainable development across the four groups of countries, giving each one practical guidance on how to implement these priorities.

\section{The priority areas and practical measures for greening and sustainable development of the OECD countries and Ukraine}

The results of calculating the median value of the GI over the 1990-2018 period for 14 OECD countries and Ukraine using the above method are presented in Table 3.

To determine the goals and priorities for greening and sustainable development for the four groups of countries, we developed a matrix based on the index method presented in Figure 2. 
Priorities for Greening and the Sustainable Development of OECD Member Countries and Ukraine...

\begin{tabular}{|c|c|c|c|c|c|c|c|c|c|c|c|c|c|c|c|}
\hline 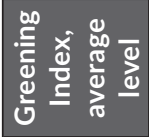 & $\begin{array}{l}a \\
\dot{q} \\
\dot{v}\end{array}$ & \begin{tabular}{l}
$\circ$ \\
\multirow{5}{0}{} \\
$\dot{0}$
\end{tabular} & 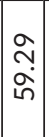 & $\begin{array}{l}\stackrel{\sim}{ } \\
\infty \\
\infty \\
\text { in }\end{array}$ & î. & 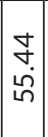 & 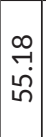 & 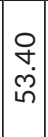 & $\mid$\begin{tabular}{l|}
$\infty$ \\
$\infty$ \\
$i$ \\
$i n$
\end{tabular} & 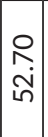 & $\begin{array}{l}\hat{0} \\
\text { กิ } \\
\text { ก̂. }\end{array}$ & $\begin{array}{l}\text { 오 } \\
\stackrel{1}{1} \\
\text { in }\end{array}$ & \begin{tabular}{l}
0 \\
$m$ \\
$\infty$ \\
\multirow{\sigma}{*}{}
\end{tabular} & 氐 & $\begin{array}{c}\mathfrak{N} \\
\infty \\
\stackrel{N}{N}\end{array}$ \\
\hline$\hat{x}$ & $\begin{array}{l}N \\
0 \\
\end{array}$ & 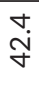 & 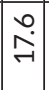 & $\begin{array}{l}\infty \\
\stackrel{\infty}{\sim} \\
\stackrel{-}{*}\end{array}$ & 옹. & مُ & 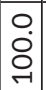 & $\begin{array}{c}0 \\
\infty \\
\end{array}$ & \begin{tabular}{l|}
$\infty$ \\
$\dot{0}$ \\
$\dot{0}$
\end{tabular} & o. & 穴 & $\begin{array}{l}n \\
\stackrel{n}{-} \\
\vec{H}\end{array}$ & $\begin{array}{l}\stackrel{+}{\infty} \\
\infty\end{array}$ & $\vec{m}$ & $\begin{array}{l}n \\
m \\
\end{array}$ \\
\hline$x^{0}$ & $\stackrel{\vec{m}}{\grave{m}}$ & $\begin{array}{l}\circ \\
\stackrel{8}{\circ} \\
\stackrel{-}{-1}\end{array}$ & \begin{tabular}{l|}
$m$ \\
$\stackrel{n}{\circ}$
\end{tabular} & $\stackrel{\circ}{+}$ & $\hat{m}$ & r. & $\begin{array}{l}\infty \\
\end{array}$ & $\stackrel{\nabla}{\sim}$ & \begin{tabular}{l|}
$\infty$ \\
$\stackrel{1}{*}$ \\
$\sim$
\end{tabular} & $\begin{array}{l}\infty \\
\stackrel{\sim}{N} \\
\end{array}$ & $\begin{array}{l}m \\
\dot{m}\end{array}$ & $\begin{array}{l}n \\
\sim \\
\sim\end{array}$ & $\stackrel{\sim}{\sim}$ & $\stackrel{\circ}{\rightarrow}$ & $\stackrel{\nabla_{i}}{\sim}$ \\
\hline$x^{n}$ & $\begin{array}{l}m \\
\dot{0} \\
\dot{0}\end{array}$ & $\begin{array}{l}a \\
a \\
\infty \\
\infty\end{array}$ & \begin{tabular}{|l|}
$\sim$ \\
$\infty$ \\
$\alpha$ \\
\end{tabular} & $\begin{array}{l}0 \\
\dot{8} \\
\text { Oे }\end{array}$ & $\begin{array}{l}+ \\
\stackrel{\sim}{\sim}\end{array}$ & 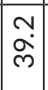 & $\begin{array}{l}+ \\
\dot{0} \\
\infty\end{array}$ & $\begin{array}{l}\infty \\
\stackrel{\infty}{\sim} \\
\sim\end{array}$ & 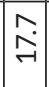 & $\hat{\alpha}$ & 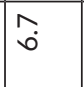 & $\begin{array}{l}10 \\
0 \\
0\end{array}$ & $\begin{array}{c}m \\
0\end{array}$ & 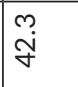 & $\begin{array}{l}0 \\
-i\end{array}$ \\
\hline$x^{ \pm}$ & $\stackrel{m}{\sim}$ & $\underset{\infty}{-1}$ & $\stackrel{-1}{+}$ & 狩 & $\hat{\vec{m}}$ & $\begin{array}{l}\hat{\sigma} \\
\infty \\
\rightarrow\end{array}$ & $\mid \begin{array}{c}0 \\
\infty \\
\dot{\sigma} \\
\end{array}$ & $\begin{array}{l}n \\
0 \\
0\end{array}$ & \begin{tabular}{|l|} 
\\
\end{tabular} & $\begin{array}{l}m \\
m \\
m\end{array}$ & \begin{tabular}{|l}
$n$ \\
0
\end{tabular} & \begin{tabular}{|c|}
$n$ \\
0
\end{tabular} & $\stackrel{+}{\circ}$ & O̊ & $\stackrel{m}{0}$ \\
\hline$x^{\infty}$ & ণ্ল & in & $\begin{array}{l}\llcorner \\
\llcorner\end{array}$ & 으 & 品 & $\stackrel{\mathcal{F}}{\sim}$ & $\hat{\sigma}$ & ○) & $\stackrel{1}{\wedge}$ & 于 & ā & ষ্ & 억 & ळे & $\stackrel{0}{\sim}$ \\
\hline$x^{n}$ & 어 & in & in & రె & in & N & $\underset{\sim}{\mathscr{\sim}}$ & $\approx$ & \& & $\hat{0}$ & 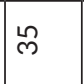 & 2 & ঠ & 六 & $\stackrel{\curvearrowright}{\rightarrow}$ \\
\hline$x^{-5}$ & \begin{tabular}{|l|} 
\\
\\
\\
\end{tabular} & î & 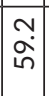 & 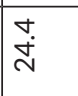 & $\begin{array}{l}\hat{\infty} \\
\infty \\
\infty\end{array}$ & $\begin{array}{c}m \\
\text { in }\end{array}$ & $\begin{array}{l}\hat{i} \\
\dot{\circ}\end{array}$ & 壬 & $\begin{array}{l}\stackrel{N}{N} \\
\stackrel{m}{n}\end{array}$ & \begin{tabular}{|c|} 
\\
$\vec{b}$ \\
\end{tabular} & $\begin{array}{l}\infty \\
\stackrel{\infty}{\text { m }}\end{array}$ & $\begin{array}{l}a \\
\infty \\
i \\
\sim\end{array}$ & $\begin{array}{l}-1 \\
\infty \\
m\end{array}$ & $\begin{array}{l}m \\
\dddot{m} \\
\infty\end{array}$ & $\begin{array}{l}m \\
0 \\
\dot{m}\end{array}$ \\
\hline$x^{0}$ & $\stackrel{m}{\sim}$ & 告 & $\begin{array}{ll} \\
0 \\
\end{array}$ & $\stackrel{m}{N}$ & $\begin{array}{l}0 \\
\stackrel{0}{r}\end{array}$ & $\stackrel{\text { Nִ }}{N}$ & 우 & $\begin{array}{l}m \\
0\end{array}$ & $\begin{array}{l}\dot{v} \\
\dot{m}\end{array}$ & \begin{tabular}{|l|} 
\\
$\dot{m}$
\end{tabular} & $\stackrel{\sigma}{\rightarrow}$ & \begin{tabular}{|c|}
0 \\
$\stackrel{1}{-}$ \\
\end{tabular} & $\stackrel{a}{0}$ & 웅 & $\stackrel{\sigma}{\vec{N}}$ \\
\hline$x^{0}$ & 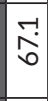 & 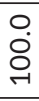 & $\begin{array}{l}\text { Na } \\
\text { No } \\
\end{array}$ & ָָ & $\begin{array}{l}\stackrel{n}{\sim} \\
\stackrel{f}{f}\end{array}$ & 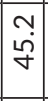 & $\begin{array}{l}m \\
\stackrel{m}{\rho} \\
\end{array}$ & 高 & $\begin{array}{l}0 \\
\dot{0} \\
\sim\end{array}$ & \begin{tabular}{|c|} 
\\
$\dot{b}$ \\
-
\end{tabular} & Nָ & $\stackrel{2}{\wedge}$ & $\begin{array}{l}0 \\
\stackrel{0}{0}\end{array}$ & $\begin{array}{l}\forall \\
0 \\
\rightarrow-1\end{array}$ & 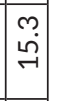 \\
\hline$x^{\infty}$ & N & 욱 & $\underset{\sim}{\sim}$ & $\infty$ & $\stackrel{N}{N}$ & ○ & in & in & $\begin{array}{l}\infty \\
0\end{array}$ & 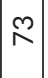 & 2 & $\stackrel{\infty}{\alpha}$ & $\hat{\infty}$ & $\stackrel{\text { I }}{+}$ & in \\
\hline$\vec{x}$ & \begin{tabular}{|c|} 
\\
$\infty$ \\
$\infty$ \\
\end{tabular} & กึ & $\stackrel{4}{\sim}$ & $\begin{array}{l}\infty \\
\text { in } \\
\text { in }\end{array}$ & 공 & 官 & ㄱ. & $\begin{array}{l}0 \\
\dot{m} \\
m\end{array}$ & 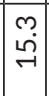 & \begin{tabular}{|l|}
$n$ \\
$\dot{m}$
\end{tabular} & $\stackrel{\infty}{\stackrel{N}{N}}$ & \begin{tabular}{|l|} 
\\
\\
\\
\end{tabular} & $\stackrel{\wedge}{\wedge}$ & $\begin{array}{l}\hat{y} \\
\dot{\sigma}\end{array}$ & $\stackrel{N}{N}$ \\
\hline$x^{0}$ & \begin{tabular}{|l|}
\multirow{2}{*}{} \\
î. \\
\end{tabular} & 穴 & \begin{tabular}{|l|} 
\\
$\infty$ \\
$\infty$ \\
$\infty$
\end{tabular} & ผू & $\begin{array}{l}0 \\
\stackrel{1}{R}\end{array}$ & $\begin{array}{l}N \\
\text { in } \\
\infty\end{array}$ & $\begin{array}{c}\overrightarrow{1} \\
\infty \\
\infty \\
0\end{array}$ & 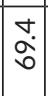 & $\begin{array}{l}\infty \\
\dot{\square} \\
\infty\end{array}$ & 今. & 옹 & $\begin{array}{l}0 \\
\dot{j} \\
\dot{v}\end{array}$ & $\begin{array}{l}\circ \\
\stackrel{\infty}{\infty}\end{array}$ & $\stackrel{m}{N}$ & $\begin{array}{l} \\
\dot{f} \\
\dot{f}\end{array}$ \\
\hline$x^{n}$ & 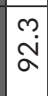 & m. & 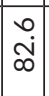 & $\begin{array}{l}\checkmark \\
\infty \\
\infty \\
\infty\end{array}$ & $\stackrel{\wedge}{a}$ & 虫 & ले & $\begin{array}{c}0 \\
\dot{i} \\
\infty\end{array}$ & 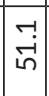 & \begin{tabular}{|l|} 
\\
0 \\
0 \\
0
\end{tabular} & 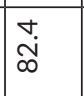 & 今. & $\begin{array}{l}0 \\
0 \\
0 \\
\end{array}$ & N & \begin{tabular}{l|l|}
$\infty$ \\
$\infty$ \\
\end{tabular} \\
\hline$x^{t}$ & बे & ㅇ. & 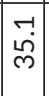 & ir & 여 & $\begin{array}{l}n \\
0 \\
0 \\
0\end{array}$ & $\begin{array}{l}n \\
\\
\end{array}$ & 紊 & $\begin{array}{l}0 \\
0 \\
\end{array}$ & \begin{tabular}{|c|} 
\\
0 \\
0 \\
0
\end{tabular} & $\begin{array}{l}0 \\
\dot{f} \\
\dot{f}\end{array}$ & 守 & $\begin{array}{l}\hat{D} \\
m\end{array}$ & $\begin{array}{l}0 \\
\tilde{N} \\
\text { in }\end{array}$ & 竎 \\
\hline$x^{\infty}$ & \begin{tabular}{|l|}
$n$ \\
$\dot{\alpha}$ \\
\end{tabular} & $\begin{array}{l}\stackrel{ナ}{+} \\
\stackrel{+}{N}\end{array}$ & \begin{tabular}{|l|} 
\\
\\
\\
\end{tabular} & $\stackrel{\sim}{\sim}$ & à & $\stackrel{\sim}{\sim}$ & $\begin{array}{c}+ \\
\infty \\
\dot{\sigma} \\
\dot{\gamma}\end{array}$ & $\begin{array}{c}\stackrel{N}{r} \\
\dot{\gamma}\end{array}$ & $\begin{array}{l}0 \\
\text { î. } \\
\text { o }\end{array}$ & \begin{tabular}{|l|}
$\infty$ \\
लె. \\
\end{tabular} & 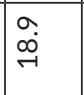 & \begin{tabular}{|l|}
$\stackrel{m}{\omega}$ \\
$\stackrel{2}{N}$
\end{tabular} & $\begin{array}{l}\hat{\sigma} \\
0 \\
\sigma\end{array}$ & 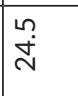 & ১े. \\
\hline$x^{2}$ & \begin{tabular}{l}
\multirow{N}{*}{} \\
\end{tabular} & $\begin{array}{l}0 \\
\stackrel{g}{\sigma}\end{array}$ & \begin{tabular}{|l|}
\multirow{r}{*}{} \\
$\stackrel{\sim}{\sim}$ \\
\end{tabular} & $\begin{array}{c}\hat{N} \\
\infty \\
\infty\end{array}$ & 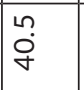 & $\begin{array}{l}\vec{r} \\
\dot{0} \\
\text { in }\end{array}$ & $\begin{array}{l}7 \\
\dot{b} \\
\text { in }\end{array}$ & $\begin{array}{l} \\
\dot{j} \\
\dot{v}\end{array}$ & $\begin{array}{l} \\
\stackrel{\infty}{m} \\
\end{array}$ & \begin{tabular}{|l|}
$n$ \\
$\infty$ \\
$\infty$ \\
\end{tabular} & \begin{tabular}{|l|} 
\\
$\infty$ \\
$\infty$ \\
$i n$
\end{tabular} & \begin{tabular}{|c|}
0 \\
$\dot{N}$ \\
\end{tabular} & ন্். & 웅 & $\begin{array}{l}n \\
\dot{m} \\
\end{array}$ \\
\hline$x^{-1}$ & 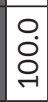 & 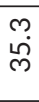 & \begin{tabular}{|l|} 
\\
0 \\
0 \\
0
\end{tabular} & $\begin{array}{l}\text { O } \\
\stackrel{D}{N}\end{array}$ & $\begin{array}{l}0 \\
\infty \\
\infty \\
i n\end{array}$ & 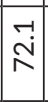 & \begin{tabular}{|c|}
0 \\
$\dot{q}$ \\
\end{tabular} & $\hat{\hat{i}}$ & \begin{tabular}{|l|}
$n$ \\
\\
\\
\end{tabular} & \begin{tabular}{|l|}
$m$ \\
$\dot{H}$ \\
\end{tabular} & ্ָ & 今े & $\underset{f}{\stackrel{-}{f}}$ & O̊. & $\begin{array}{l}\infty \\
\dot{m} \\
\stackrel{\sim}{\sim}\end{array}$ \\
\hline & $\begin{array}{l}\frac{c}{2} \\
0 \\
0 \\
\vdots \\
3 \\
n\end{array}$ & $\begin{array}{l}\frac{c}{\pi} \\
\frac{2}{\pi} \\
\frac{2}{2}\end{array}$ & 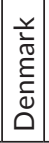 & 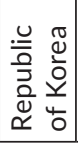 & 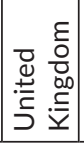 & \begin{tabular}{|c|} 
\\
$\stackrel{y}{0}$ \\
$\frac{\pi}{\pi}$ \\
$\frac{\pi}{4}$ \\
\end{tabular} & 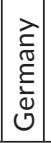 & 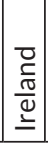 & 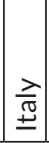 & $\begin{array}{l}\cdot \frac{c}{\pi} \\
\frac{2}{2} \\
\end{array}$ & 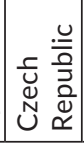 & $\begin{array}{l}0 \\
\cdot \frac{u}{x} \\
\frac{0}{2} \\
\end{array}$ & $\begin{array}{l}\frac{1}{1} \\
\sqrt[0]{0} \\
00 \\
5 \\
3 \\
1 \\
\end{array}$ & 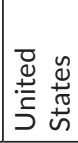 & 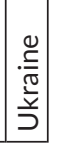 \\
\hline
\end{tabular}




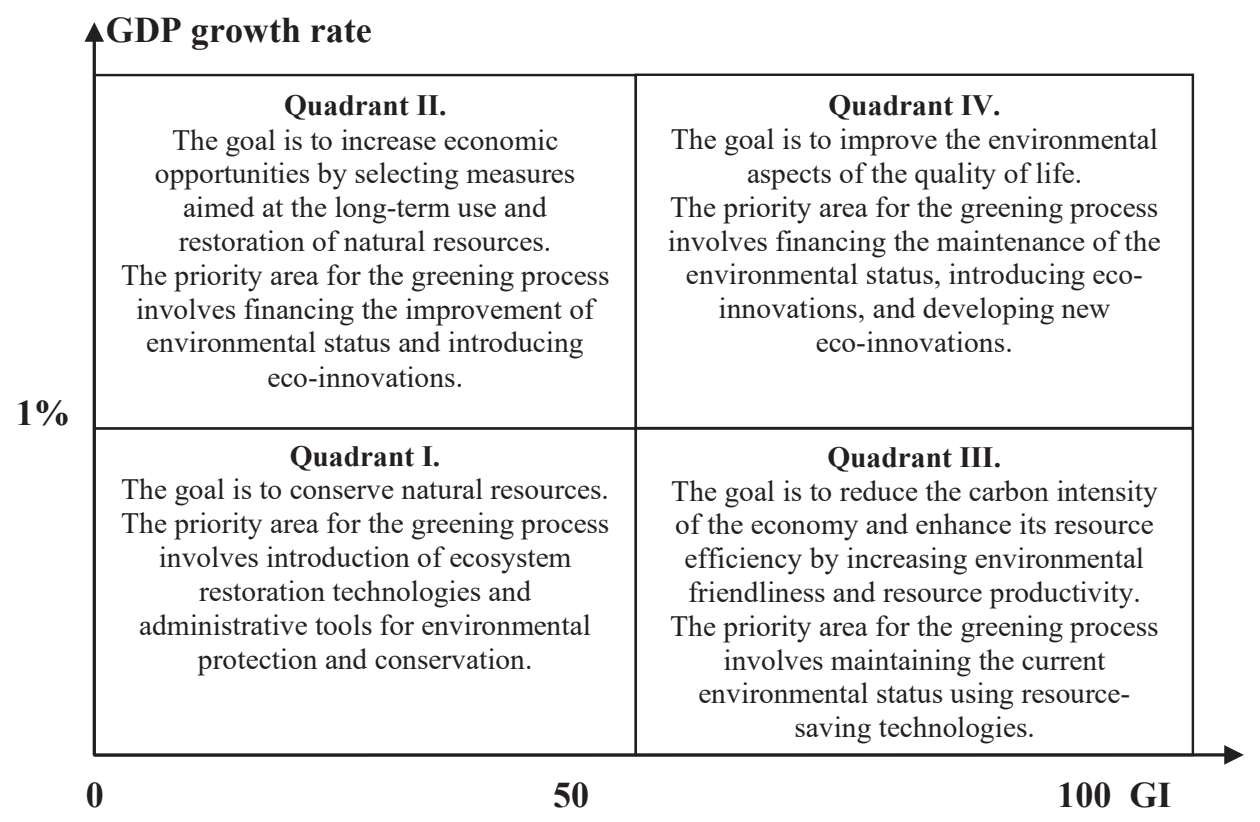

Figure 2. The matrix for identifying priority areas for greening and sustainable development Source: authors' own elaboration based on Yashalova (2015).

Quadrant I includes countries with a GI value of less than 50.0 and an average annual GDP growth rate of up to $1 \%$, thus specifying the introduction of ecosystem restoration technologies and administrative tools for environmental conservation as their priorities for the greening process. Quadrant II encompasses countries with a GI value of less than 50.0 and an average annual GDP growth rate of more than $1 \%$, which calls for financing environmental improvements and introduction of eco-innovations. Quadrant III includes countries with a GI value of over 50.0, and an average annual GDP growth rate of less than $1 \%$. Their priority area for the greening process is maintaining the current environmental status using resource-saving technologies. The countries falling into quadrant IV - with a GI value of more than 50.0 and an average annual GDP growth rate of more than $1 \%$ - are advised to finance the maintenance of their environmental status, introduce eco-innovations and develop new eco-innovations.

In our opinion, the main measures for the implementation of the priority areas for greening and sustainable development should primarily focus on introducing incentives to increase resource and natural asset efficiency. This will contribute to improving productivity, spreading eco-innovations, opening new markets for eco-products, attracting investors, and improving socio-economic stability. It should include promoting efficient resource management and the reduction of waste and energy consumption. It reflects not only how much energy is utilized in the economy but also the changes in energy consumption across sectors (Setyawan 2020, p. 394). It will thus ex- 
pand the range of eco-innovation opportunities by taking certain political measures and developing a framework to spread new production technologies aimed at addressing environmental issues. It will also create new international markets for eco-products by stimulating demand for environmentally-friendly technologies, products and services, and increase employment opportunities in the environmental field in different countries.

At the same time, it is necessary to understand that all these measures should be implemented in the context of increasingly scarce and deteriorating resources scarcity, as well as the increasing cost of investment and capital intensity of infrastructure. Therefore, the problems that arise in the process of greening and sustainable development should be solved only using the innovative potential of the modern economy. It is viewed as a country's set of scientific, technological, financial, economic, industrial, social, cultural, and educational capacities in the field of environmental protection and restoration, the efficient use of natural resources, improving productivity in the environmental field, and reducing resource intensity required to ensure sustainable development.

That is why, in our view, implementing the identified priority areas for greening and sustainable development requires taking both economic and environmental measures, including:

1. Economic measures:

- increasing GDP, enhancing production efficiency, and creating new improved products to be used as a material basis for the development of the greening process;

- implementing structural changes in the economy that lead to the emergence of new sectors, products, and services and that can eliminate the problem of technological deadlocks, especially regarding infrastructure;

- ensuring financial consolidation by reviewing the composition and efficiency of public expenditure and raising revenues with adequate pollution charges, among others;

- ensuring investor confidence by improving the predictability and stability of public policy on key development and environmental issues;

- ensuring balanced macroeconomic conditions and stable resource prices;

- economic diversification, reducing environmental impact, and mitigating natural hazards/risk management by means of eco-innovation;

- introducing innovative and resource-efficient technologies of production and consumption, and the use of "green" technologies;

- raising incomes and the quality of life, reducing inequities in access to natural resources.

2. Environmental measures:

- regular monitoring of the environmental status;

- introducing ecosystem and biodiversity restoration technologies; 
- increasing the productivity and efficiency of natural resource use and waste management;

- energy saving thanks to closed cycle manufacturing;

- improving the use of natural and other types of capital within environmental limits with the help of non-renewable natural capital.

Based on the Greening Index calculation and comparing it with the GDP growth rate, we have classified all the analyzed countries into four quadrants. We have also provided practical guidance on implementing priority areas for greening and sustainable development (Table 4).

Table 4. Classification of countries based on the calculations of their greening index and average GDP growth rate and guidance on implementing priority areas for greening and sustainable development

\begin{tabular}{|c|c|c|}
\hline $\begin{array}{c}\text { Priority areas for } \\
\text { greening }\end{array}$ & Countries & Practical guidance \\
\hline $\begin{array}{l}\text { Quadrant I. } \\
\text { Implementing eco- } \\
\text { system restoration } \\
\text { technologies and } \\
\text { administrative tools } \\
\text { for environmental } \\
\text { conservation }\end{array}$ & Ukraine & $\begin{array}{l}\text { - regularly monitor the environment status; } \\
\text { - introduce ecosystem and biodiversity restoration technolo- } \\
\text { gies; } \\
\text { - ensure financial consolidation by reviewing the composition } \\
\text { and efficiency of public expenditure and raising revenues } \\
\text { with adequate pollution charges, among others; } \\
\text { - ensure investor confidence by improving the predictability } \\
\text { and stability of public policy on key development and envi- } \\
\text { ronmental issues; } \\
\text { - ensure balanced macroeconomic conditions and stable re- } \\
\text { source prices; } \\
\text { - reduce environmental impact and natural hazards mitigation/ } \\
\text { risk management }\end{array}$ \\
\hline $\begin{array}{l}\text { Quadrant II . } \\
\text { Financing improve- } \\
\text { ment of environ- } \\
\text { mental status } \\
\text { and introduction } \\
\text { of eco-innovations }\end{array}$ & $\begin{array}{l}\text { Hungary, } \\
\text { United } \\
\text { States }\end{array}$ & $\begin{array}{l}\text { - introduce ecosystem and biodiversity conservation technol- } \\
\text { ogies; } \\
\text { - raise revenues with adequate pollution charges; } \\
\text { - introduce innovations and structural changes in the econo- } \\
\text { my that lead to the emergence of new sectors, products, and } \\
\text { services and that can eliminate the problem of technological } \\
\text { deadlocks, especially concerning infrastructure; } \\
\text { - ensure investor confidence by improving the predictability } \\
\text { and stability of public policy on key development and envi- } \\
\text { ronmental issues; } \\
\text { - ensure balanced macroeconomic conditions and stable re- } \\
\text { source prices; } \\
\text { - reduce environmental impact and mitigate natural hazards/ } \\
\text { risk management }\end{array}$ \\
\hline $\begin{array}{l}\text { Quadrant III . } \\
\text { Maintaining the } \\
\text { current environ- } \\
\text { mental status using } \\
\text { resource-saving } \\
\text { technologies }\end{array}$ & Italy & $\begin{array}{l}\text { - regularly monitor the environment status; } \\
\text { - introduce ecosystem and biodiversity restoration technolo- } \\
\text { gies; } \\
\text { - introduce innovative and resource-efficient technologies } \\
\text { of production and consumption, use «green» technologies; } \\
\text { - reduce environmental impact and mitigate natural hazards/ } \\
\text { risk management }\end{array}$ \\
\hline
\end{tabular}




\begin{tabular}{|c|c|c|}
\hline $\begin{array}{c}\text { Priority areas for } \\
\text { greening }\end{array}$ & Countries & Practical guidance \\
\hline $\begin{array}{l}\text { Quadrant IV . } \\
\text { Financing mainte- } \\
\text { nance of the en- } \\
\text { vironmental sta- } \\
\text { tus, introduction } \\
\text { of eco-innovations } \\
\text { and development } \\
\text { of new eco-inno- } \\
\text { vations }\end{array}$ & $\begin{array}{l}\text { Sweden, } \\
\text { Japan, } \\
\text { Denmark, } \\
\text { Republic } \\
\text { of Korea, } \\
\text { United } \\
\text { Kingdom, } \\
\text { France, } \\
\text { Germany, } \\
\text { Ireland, } \\
\text { Spain, } \\
\text { Czech } \\
\text { Republic, } \\
\text { Mexico }\end{array}$ & $\begin{array}{l}\text { - increase productivity and the efficiency of natural resource } \\
\text { use; } \\
\text { - improve the use of natural and other types of capital within } \\
\text { environmental limits with the help of non-renewable natural } \\
\text { capital; } \\
\text { - raise funding for environmental research and eco-innovation; } \\
\text { - economic diversification, i.e., improve economic risk manage- } \\
\text { ment by introducing eco-innovation; } \\
\text { - introduce innovative and resource-efficient technologies } \\
\text { of production and consumption, use "green» technologies; } \\
\text { - reduce environmental impact and mitigate natural hazards/ } \\
\text { risk management }\end{array}$ \\
\hline
\end{tabular}

Source: author's development.

Thus, Ukraine falls into quadrant I, with an average GI value of 22.82 and an average annual GDP growth rate of $0.3 \%$ between 1990 and 2017. Quadrant II includes two of the studied countries: Hungary (GI - 48.36, average annual GDP growth rate $-2.0 \%$ ) and the USA (GI - 44.12, average annual GDP growth rate $-2.4 \%$ ). Quadrant III includes Italy (GI -52.86 , average annual GDP growth rate $-0.7 \%$ ). Finally, quadrant IV encompasses most of the analyzed countries: Sweden (GI - 76.49, average annual GDP growth rate - 2.65\%), Japan (GI - 64.96, average annual GDP growth rate $-1.9 \%$ ), Denmark (GI - 59.29, average annual GDP growth rate $-1.85 \%$ ), Republic of Korea (GI - 58.20, average annual GDP growth rate - 4.55\%), UK (GI - 57.71, average annual GDP growth rate - 2.0\%), France (GI - 55.44, average annual GDP growth rate $-1.7 \%$ ), Germany (GI - 55.18, average annual GDP growth rate $-2.37 \%$ ), Ireland (GI - 53.4, average annual GDP growth rate - 7.0\%), Spain (GI - 52.7, average annual GDP growth rate - 2\%), the Czech Republic (GI - 52.67, average annual GDP growth rate $-1.67 \%$ ), Mexico (GI - 51.5, average annual GDP growth rate $-2.58 \%$ ).

We believe that to conserve natural resources, the priorities for greening and sustainable development in Ukraine should include the introduction of ecosystem restoration technologies and administrative tools for environmental conservation through regular environmental monitoring. It should also ensure financial consolidation by reviewing the composition and efficiency of public expenditure and increase revenues with adequate pollution charges. Additionally, it should ensure investor confidence by improving the predictability and stability of public policy on key development and environmental issues, ensuring balanced macroeconomic conditions and stable resource prices. Finally, it should reduce environmental impact and mitigate natural hazards/risk management.

Regarding Hungary and United States, the main goal is to increase economic opportunities by selecting measures for the long-term use and restoration of natural resources. Therefore, the priority areas for greening should include financing the environmental status improvement and introducing eco-innovations. This can be done 
by implementing ecosystem and biodiversity conservation technologies; raising revenues with adequate pollution charges; introducing innovations and structural changes in the economy that lead to the emergence of new sectors, products, and services, and that can eliminate the problem of technological deadlocks, especially regard infrastructure; ensuring investor confidence by improving the predictability and stability of public policy on key development and environmental issues; ensuring balanced macroeconomic conditions and stable resource prices; and reducing environmental impact and mitigating natural hazards/risk management.

For Italy, the main goal is to reduce the carbon intensity of the economy and enhance its resource efficiency by increasing environmental friendliness and resource productivity. This means that its priority areas for greening should include maintaining the current environmental status with resource-saving technologies through regular environmental monitoring, introducing ecosystem and biodiversity restoration technologies, introducing innovations, and using "green" technologies, as well as reducing environmental impact and mitigating natural hazards/risk management.

Finally, with a view to improving the environmental aspects of the quality of life in countries such as Sweden, Japan, Denmark, the Republic of Korea, the UK, France, Germany, Ireland, Spain, the Czech Republic, and Mexico, the priorities of greening and sustainable development include financing the maintenance of the environmental status, introducing eco-innovation and developing new eco-innovations by increasing the productivity and efficiency of natural resource use, improving the use of natural and other types of capital within environmental limits using non-renewable natural capital, raising funding for environmental research and eco-innovation, economic diversification, i.e., improving economic risk management with introducing eco-innovation, introducing innovative and resource-efficient technologies of production and consumption, using "green" technologies, and reducing environmental impact and mitigating natural hazards/risk management.

Thus, the priority areas for greening and sustainable development and the measures for implementing them should match both their current environmental status and the level of economic development. Finally, effective and strict international environmental protection laws should be adopted worldwide (Singh et al. 2019, p. 87). The European financial market, whose participants are mainly from developed countries, has already begun to implement environmentally friendly regulations (Janicka 2016, p. 35).

\section{Conclusion}

Identifying priority areas for greening and sustainable development must be based on a reliable environmental strategy primarily aimed at improving the current state of the global ecological and economic space. The global system of strategic goals of greening and sustainable development has a hierarchical structure. The upper level features a system of global greening goals, the second level includes a system of national goals, and the third level contains a system of goals in specific areas of green- 
ing. Although global greening goals are relevant to all countries, they must be tailored to the regional and national specificities, as well as each country's level of economic development, which was stated as the research purpose and that was proved as a result of the study.

Our findings could have direct implications in terms of countries' ecological policies. Based on our analysis, we have determined and justified the following priority areas for greening and sustainable development and measures for their implementation for four groups of the countries:

- for Sweden, Japan, Denmark, the Republic of Korea, the UK, France, Germany, Ireland, Spain, the Czech Republic, and Mexico, where it is necessary to improve environmental aspects of the quality of life, these priorities should include financing to maintain the environmental status, introducing eco-innovation and developing new eco-innovations by increasing the productivity and efficiency of natural resource use, and raising funding for environmental research and eco-innovation;

- for Italy, whose main goal is to reduce the carbon intensity of the economy and enhance its resource efficiency by increasing environmental friendliness and resource productivity, the priority areas for greening should include maintaining the current environmental status with resource-saving technologies by introducing ecosystem and biodiversity restoration technologies, introducing innovations, and using "green" technologies;

- for Hungary and the USA, to increase economic opportunities by selecting measures aimed at the long-term use and restoration of natural resources, the priorities for greening should include financing environmental improvements, introducing eco-innovations with ecosystem and biodiversity conservation technologies, and raising revenues with adequate pollution charges;

- for Ukraine, which aims to conserve natural resources, the priority areas for greening should include introducing ecosystem restoration technologies and administrative tools for environmental conservation through regular environmental monitoring, ensuring financial consolidation by reviewing the composition and efficiency of public expenditure and raising revenues with adequate pollution charges, ensuring balanced macroeconomic conditions and stable resource prices, and reducing environmental impact and mitigating natural hazards/risk management.

Based on an index method, the research allowed us to improve the hierarchical structure of the strategic goals system of the global economic space greening process. A matrix of priority directions of the greening process was then developed and practical recommendations on their implementation for different groups of countries were provided, identified after analyzing the environmental development index and GDP growth rate.

Thus, to develop a national environmentally friendly nature manage policy that ensures sustainable economic development at the national level, countries must identify 
the priority directions for sustainable development greening and find practical measures to implement them.

\section{Acknowledgements}

This research did not receive any specific grant from funding agencies in the public, commercial, or not-for-profit sectors.

\section{References}

Anderson, D. (2013), Environmental Economics and Natural Resource Management, Routledge, London, https://doi.org/10.4324/9781315884257

Anderson, K., Blackhurst, R. (1992), Effects on the environment and welfare of liberalising world trade: the cases of coal and food, The Greening of World Trade Issues, Harvester Wheatsheaf, London.

Banerjee, O., Cicowiez, M., Horridge, M., Vargas, R. (2016), A conceptual framework for integrated economic-environmental modeling, "Journal of Environment and Development”, 25 (3), pp. 276-305, https://doi.org/10.1177/1070496516658753

Blanc, I., Friot, D., Margni, M., Jolliet, O. (2008), Towards a new index for environmental sustainability based on a DALY weighting approach, "Sustainable Development", 16 (4), pp. 251-260, https://doi.org/10.1002/sd.376

Colby, M.E. (1989), The Evolution of Paradigms of Environmental Management in Development, http://documents.banquemondiale.org/curated/fr/552371468913746182 /pdf/multi-page.pdf (accessed: 26.01.2020).

Daly, H., Farley, J. (2010), Ecological economics: Principles and applications, Island Press, Washington.

Dovgal, O., Panova, I. (2018a), Environmental policy in the process of greening economic development, "Problems of Economy", 4 (38), pp. 280-285, https://doi.org/10.32 983/2222-0712-2018-4-380-385

Dovgal, O., Panova, I. (2018b), Evolution of Environmental Management Concepts in Terms of Global Environmental Challenges, "Journal of V.N. Karazin Kharkiv National University", Series: "International Relations. Economics, Country Studies. Tourism”, No. 9, pp. 109-114.

Ehrlich, P., Ehrlich, A., Holdren, J. (1977), Ecoscience: Population. Resources, Environment, W.H. Freeman and Company, San Francisco.

Esty, D. (1994), Greening the GATT: Trade, environment and the future, Peterson Institute Press, Washington.

Global Goalscast (2018), Overview, https:/globalgoalscast.org/about-us (accessed: 29.01.2020).

Green Economy Options For Ukraine (2018), Opportunities for organic agriculture, Policy Brief, http://www.green-economies-eap.org/resources/Ukraine\%20OA\%20 ENG\%2027\%20Jun.pdf (accessed: 11.02.2020). 
Green Growth Knowledge Platform (2000-2017), GGKP Data Explorer, https://www .greengrowthknowledge.org/data-explorer\#/line? country=245\&indicator=299\&st artYear=2000\&endYear=2017 (accessed: 1.03.2020).

Greening Economies in the European Union's Eastern Neighbourhood: From commitment to results. Programme (EaP GREEN) (2018), Resource Library, http://www.gre en-economies-eap.org/resources/EaP\%20GREEN_From\%20Commitments\%20to \%20Results.pdf (accessed: 12.02.2020).

Huber, J. (1991), Ecological modernization. Away from scarcity, soberness and bureaucracy, [in:] A. Mol, G. Spaargaren, A. Kalpxijk (eds.), Technologie en Milieubeheer, SDU, Den Haag.

Hull, Ch.H. (ed.) (1899), The Economic Writings of Sir William Petty, Routledge/ Thoemmes, London.

Ignatov, V., Kokin, A., Baturin, L. (1999), Balanced nature management, Rostizdat, Rostov-na-Donu.

Jackson, T. (2017), Prosperity Without Growth: Foundations for the Economy of Tomorrow, Routledge, London, https://doi.org/10.4324/9781315677453

Janicka, M. (2016), Financial markets and the challenges of sustainable growth, "Comparative Economic Research. Central and Eastern Europe”, 19 (2), pp. 27-41, https:// doi.org/10.1515/cer-2016-0011

Kazakov, N., Li, I., Popkov, V. (2009), Recreational environmental entrepreneurship. Monografiya, SPbGIEU, Sankt-Peterburg.

Kitzes, J., Peller, A., Goldfinger, S., Wackernagel, M. (2007), Current Method for Calculating National Ecological Footprint Accounts, "Science for Environment \& Sustainable Society”, 4 (1), https://pdfs.semanticscholar.org/96a3/a3a5d654008d7a0d 127e296019c90fd225fd.pdf (accessed: 11.03.2020).

Knoema Corporation (US) (2018), Gross domestic product in current prices growth rate, https://knoema.com/atlas/topics/Economy/National-Accounts-Gross-Domestic-P roduct/GDP-growth (accessed: 22.01.2020).

Malthus, T. (1798), An Essay on the Principle of Population, J. Johnson, in St. Paul's Church-Yard, London.

Matyushenko, I., Goncharenko N., Michaylova D. (2015), Future Consideration for Developing Energy Efficient Economy in Ukraine using Light Emitting Diode (LED) Enginery on the basis of NBIC-Technologies, "Global Journal of Management and Business Research: Economics and Commerce”, 15 (5), pp. 1-19, https://doi.org/10 .9734/BJEMT/2015/18567

Meadows, D., Randers, J., Meadows, D. (2007), Limits to growth. The 30-year update, Chelsea Green Publishing, White River Junction.

Meadows, D., Randers, J., Meadows, D., Behrens, W. (1972), The Limits to Growth: A Report for the Club of Rome's Project on the Predicament of Mankind, Universe Books, New York, https://doi.org/10.1349/ddlp.1

New Atlas of the Green Economy (2019), The Global Green Economy Index, https://u alcitizeninc.com/global-green-economy-index/economic-environmental-indicato rs.php?id=9 (accessed: 14.01.2020).

OECD (1990-2018), Green Growth Indicators, https://stats.oecd.org/Index.aspx?Data SetCode=GREEN_GROWTH (accessed: 5.01.2020). 
Podlesnaya, V. (2012), The formation of the economic mechanism of environmental management at the development of social reproduction socio-economic cycles, "The Mechanism of Economy Regulation", 2, pp. 208-212, http://mer.fem.sumdu.edu.ua/con tent/acticles/issue_10/V_G_PodlesnaBecoming_economic_mechanism_of_natu re_in_the_development_of_socio_economic_cycles_of_social_reproduction.pdf (accessed: 11.01.2020).

Report on Green Transformation in Ukraine. Based on OECD Green Growth Indicators (2016), http://www.green-economies-eap.org/resources/2016_GreenTransformation \%20in\%20Ukraine\%20ENG.pdf (accessed: 9.01.2020).

Reznikova, N. (2016), Ecological imperatives for extension of globalization processes: problem of economic security, "Investments: Practice and Experience", 21, pp. 23-26, http://www.investplan.com.ua/pdf/21_2016/6.pdf (accessed: 16.01.2020).

Rogers, P., Jalal, K., Boyd, J. (2006), An Introduction to Sustainable Development, Harvard University Press, Cambridge.

Setyawan, D. (2020), Economy-wide energy efficiency using a comprehensive decomposition method, "Global Journal of Environmental Science and Management", 6 (3), pp. 385-402.

Singh, A.K., Issac, J., Narayanan, K.G.S. (2019), Measurement of environmental sustainability index and its association with socio-economic indicators in selected Asian economies: an empirical investigation, "International Journal of Environment and Sustainable Development", 18 (1), https://doi.org/10.1504/IJESD.2019.098641

Sustainable Development Report (2019), Transformations to Achieve the Sustainable Development Goals, https://www.sustainabledevelopment.report (accessed: 18.01.2020).

Svenningsen, L.S., Thorsen, B.J. (2020), Preferences for Distributional Impacts of Climate Policy, "Environ Resource Economics", 75, pp. 1-24, https://doi.org/10.1007 /s10640-019-00386-z

Škrinjarić, T. (2020), Re-examining sustainable development in Europe: a data envelopment approach, "International Journal of Environment and Sustainable Development", 19 (1), pp. 72-109, https://doi.org/10.1504/IJESD.2020.10027205

United Nations (2018), Development Programme. GRID-Arendal. Global environmental trends, https://www.grida.no/resources/5488 (accessed: 21.01.2020).

United Nations (2019), United Nations Environment Programme. Global Environment Outlook 6, https://www.unenvironment.org/resources/global-environment-outlo ok-6 (accessed: 21.01.2020).

Wackernagel, M., White, K., Moran, D. (2004), Using Ecological Footprint accounts: from analysis to applications, "Environment and Sustainable Development", 3 (3/4), https://doi.org/10.1504/IJESD.2004.005077

Wackernagel, M., Lin, D., Evans, M., Hanscom, L., Raven, P. (2019), Defying the Footprint Oracle: Implications of Country Resource Trends, "Sustainability", 11, 2164, https://doi.org/10.3390/su11072164

Weizsäcker, E., Hargroves, K., Smith, M. (2010), Faktor Fünf. Die Formel für nachhaltiges Wachstum, Droemer, München.

Weizsäcker, E., Lovins, A., Lovins, L. (1995), Faktor Vier. Doppelter Wohlstand - halbierter Naturverbrauch. Der neue Bericht an den Club of Rome, Droemer Knaur, München. 
Wiesmeth, H. (2012), Environmental economics. Theory and Policy in Equilibrium, Springer, London-New York.

World Resources Institute (2018), Trends Point to Gains in Human Development, While Many Negative Human Impacts on Vital Ecosystems are Increasing, https://www.gri equity.com/resources/Environment/Global\%20Environmental\%20Trends.htm (accessed: 24.01.2020).

Yashalova, N. (2015), Assessment of the level of greening the economy of region, "Environmental economics", 3, pp. 67-75, https://www.elibrary.ru/item.asp?id=23615026 (accessed: 8.01.2020).

Yashalova, N., Ruban, D. (2016), Development of green business as an approach to financing the greening of economy, "Economic and Social Changes: Facts, Trends, Forecast", 5, pp. 219-237, https://doi.org/10.15838/esc.2016.5.47.12

Zerkalov, D. (2013), Problems of sustainable development ecology, Osnova, Kiev.

\section{Priorytety ekologizacji i zrównoważonego rozwoju krajów członkowskich OECD i Ukrainy: analiza porównawcza}

Artykuł koncentruje się na określeniu priorytetowych obszarów ekologizacji i zrównoważonego rozwoju dla krajów OECD i Ukrainy, mających na celu osiągnięcie ogólnego postępu w interakcji między gospodarką a środowiskiem, a także stworzenie koniecznych warunków wstępnych do wspierania innowacji i inwestycji w celu znalezienia nowych źródeł wzrostu gospodarczego, zgodnych z ekosystemami wykazującymi zdolność regeneracji. Udowodniono, że chociaż globalne cele gospodarki ekologicznej są istotne dla wszystkich krajów świata, muszą być dostosowane do specyfiki regionalnej i krajowej, a także do poziomu rozwoju gospodarczego każdego kraju.

W badaniu wykorzystano ogólne jakościowe i ilościowe metody badań ekonomicznych, w tym analizę systematyczną, porównawczą, metody analizy logicznej i statystycznej, metodę wskaźnikową i inne. Zastosowane podejście teoretyczne i metodologiczne pozwoliło zidentyfikować ogólne trendy rozwoju czynników środowiskowych w krajach OECD i na Ukrainie oraz ich wpływ na wzrost gospodarczy. W badaniu przeanalizowano w szczególności obecny stan i perspektywy ekologizacji i zrównoważonego rozwoju w krajach OECD i na Ukrainie w oparciu o wyniki kompleksowej oceny poziomu ekologizacji tych gospodarek oraz relacji między wewnętrznym środowiskiem ekologicznym - ocenianym za pomocą wskaźników „zielonego wzrostu” - a ich rozwojem gospodarczym. Artykuł uzasadnia również wprowadzenie priorytetów w zakresie ekologizacji i zrównoważonego rozwoju, a także sugeruje praktyczne środki ich realizacji. Mogą one służyć jako podstawa do opracowania polityki skutecznego zarządzania środowiskiem i opracowania krajowego systemu zarządzania i administracji przyjaznego środowisku.

Słowa kluczowe: globalne problemy środowiskowe, globalna strategia środowiskowa, zielony wzrost, ekologizacja, priorytety ekologizacji

i zrównoważonego rozwoju 